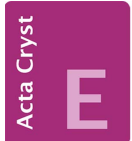

ISSN 2056-9890

\section{Crystal structure study of a cobaltoan dolomite from Kolwezi, Democratic Republic of Congo}

\section{Natale Perchiazzi}

Earth Sciences Department, Pisa University, Via S. Maria 53, I-56126, Pisa, Italy.

*Correspondence e-mail: natale.perchiazzi@unipi.it

Received 4 February 2015; accepted 13 February 2015

Edited by I. D. Brown, McMaster University, Canada

A structural study has been undertaken on a cobaltoan dolomite, with chemical formula $\mathrm{CaMg}_{0.83} \mathrm{Co}_{0.17}\left(\mathrm{CO}_{3}\right)_{2}$ (calcium magnesium cobalt dicarbonate), from Kolwezi, Democratic Republic of Congo. Pale-pink euhedral cobaltoan dolomite was associated with kolwezite $\left[\left(\mathrm{Cu}_{1.33} \mathrm{Co}_{0.67}\right)\right.$ $\left.\left(\mathrm{CO}_{3}\right)(\mathrm{OH})_{2}\right]$ and cobaltoan malachite $\left[(\mathrm{Cu}, \mathrm{Co})_{2}\left(\mathrm{CO}_{3}\right)-\right.$ $(\mathrm{OH})_{2}$ ]. A crystal with a Co:Mg ratio of 1:5.6 (SEM/EDAX measurement), twinned on (11 $\overline{2} 0)$ was used for crystal structural refinement. The refinement of the structural model of Reeder \& Wenk [Am. Mineral. (1983), 68, 769-776; Ca at site $3 a$ with site symmetry $\overline{3}$; $\mathrm{Mg}$ site at site $3 b$ with site symmetry $\overline{3} ; \mathrm{C}$ at site $6 c$ with site symmetry $3 ; \mathrm{O}$ at site $18 f$ with site symmetry 1] showed that Co is totally incorporated in the $\mathrm{Mg}$ site, with refined occupancy $\mathrm{Mg}_{0.83} \mathrm{Co}_{0.17}$, which compares with $\mathrm{Mg}_{0.85} \mathrm{Co}_{0.15}$ from chemical data. The Co substitution reflects in the expansion of the cell volume, with a pronounced increasing of the $c$ cell parameter.

Keywords: crystal structure; dolomite; cobaltoan; Kolwezi.

CCDC reference: 1049359

\section{Related literature}

For general background, see: Barton et al. (2015); Pertlik (1986). For isotypic structures, see: Reeder \& Wenk (1983). For kolwezite, see: Deliens \& Piret (1980).

\section{Experimental}

\subsection{Crystal data}

$\mathrm{CaMg}_{0.83} \mathrm{Co}_{0.17}\left(\mathrm{CO}_{3}\right)_{2}$

$$
M_{r}=190.38
$$

Trigonal, $R \overline{3}$
$a=4.8158(1) \AA$
$c=16.0488(6) \AA$
$V=322.34(2) \AA^{3}$
$Z=3$

\subsection{Data collection \\ Bruker SMART Breeze CCD diffractometer \\ Absorption correction: multi-scan (SADABS; Bruker, 2008) \\ $T_{\min }=0.621, T_{\max }=0.746$}

\subsection{Refinement}

$R\left[F^{2}>2 \sigma\left(F^{2}\right)\right]=0.019$

$w R\left(F^{2}\right)=0.059$

$S=0.96$

258 reflections

Table 1

Selected bond lengths $(\AA)$.

\begin{tabular}{llll}
\hline $\mathrm{Ca} 1-\mathrm{O} 1^{\mathrm{i}}$ & $2.3833(10)$ & $\mathrm{C} 1-\mathrm{O} 1$ & $1.2853(9)$ \\
$(\mathrm{Mg} 1 / \mathrm{Co} 1)-\mathrm{O} 1^{\mathrm{ii}}$ & $2.0923(9)$ & & \\
\hline
\end{tabular}

Symmetry codes: (i) $-x+y+\frac{1}{3},-x+\frac{2}{3}, z-\frac{1}{3}$; (ii) $-x+y+\frac{2}{3},-x+\frac{1}{3}, z+\frac{1}{3}$.

Data collection: APEX2 (Bruker, 2008); cell refinement: SAINT (Bruker, 2008); data reduction: $S A I N T$; program(s) used to solve structure: SHELXS2014 (Sheldrick, 2008); program(s) used to refine structure: SHELXL2014 (Sheldrick, 2008, 2015) and WinGX (Farrugia, 2012); molecular graphics: CrystalMaker (CrystalMaker, 2010); software used to prepare material for publication: publCIF (Westrip, 2010).

\section{Acknowledgements}

Dr H. Goethals, Royal Belgian Institute for Natural Sciences, is kindly acknowledged for providing the mineral sample.

Supporting information for this paper is available from the IUCr electronic archives (Reference: BR2247).

\section{References}

Barton, I., Yang, H. \& Barton, M. D. (2015). Can. Mineral.. In the press. Bruker (2008). APEX2, SAINT and SADABS. Bruker AXS Inc. Madison, Wisconsin, USA.

CrystalMaker (2010). CrystalMaker. CrystalMaker Software Ltd, Yarnton, England.

Deliens, M. \& Piret, P. (1980). Bull. Mineral. 103, 179-184.

Farrugia, L. J. (2012). J. Appl. Cryst. 45, 849-854.

Pertlik, F. (1986). Acta Cryst. C42, 4-5.

Reeder, R. J. \& Wenk, H. R. (1983). Am. Mineral. 68, 769-776.

Sheldrick, G. M. (2008). Acta Cryst. A64, 112-122.

Sheldrick, G. M. (2015). Acta Cryst. C71, 3-8.

Westrip, S. P. (2010). J. Appl. Cryst. 43, 920-925. 


\section{supporting information}

Acta Cryst. (2015). E71, i3 [doi:10.1107/S2056989015003126]

\section{Crystal structure study of a cobaltoan dolomite from Kolwezi, Democratic Republic of Congo}

\section{Natale Perchiazzi}

\section{S1. Synthesis and crystallization}

Cobaltoan dolomite was picked from a kolwezite sample from Kolwezi (inventory number RC 3987) kindly provided us by H. Goethals, Royal Belgian Institute for Natural Sciences, Brussels. Pale pink euhedral cobaltoan dolomite was associated with kolwezite and cobaltoan malachite. All these minerals occur in the supergene zones of $\mathrm{Cu}-\mathrm{Co}$ sulfide ore deposits,originating from the alteration of primary sulphides such as carrollite, $\mathrm{Cu}(\mathrm{Co}, \mathrm{Ni})_{2} \mathrm{As}_{4}$

\section{S2. Refinement}

During the refinement, the twinning according to the $(11 \overline{2} 0)$ common law was detected and accounted for, with a refined BASF parameter of 0.798 . The sum of $\mathrm{Co}$ and $\mathrm{Mg}$ occupancies in $\mathrm{Mg}$ site was constrained to be equal to 1, no other constraint was applied.

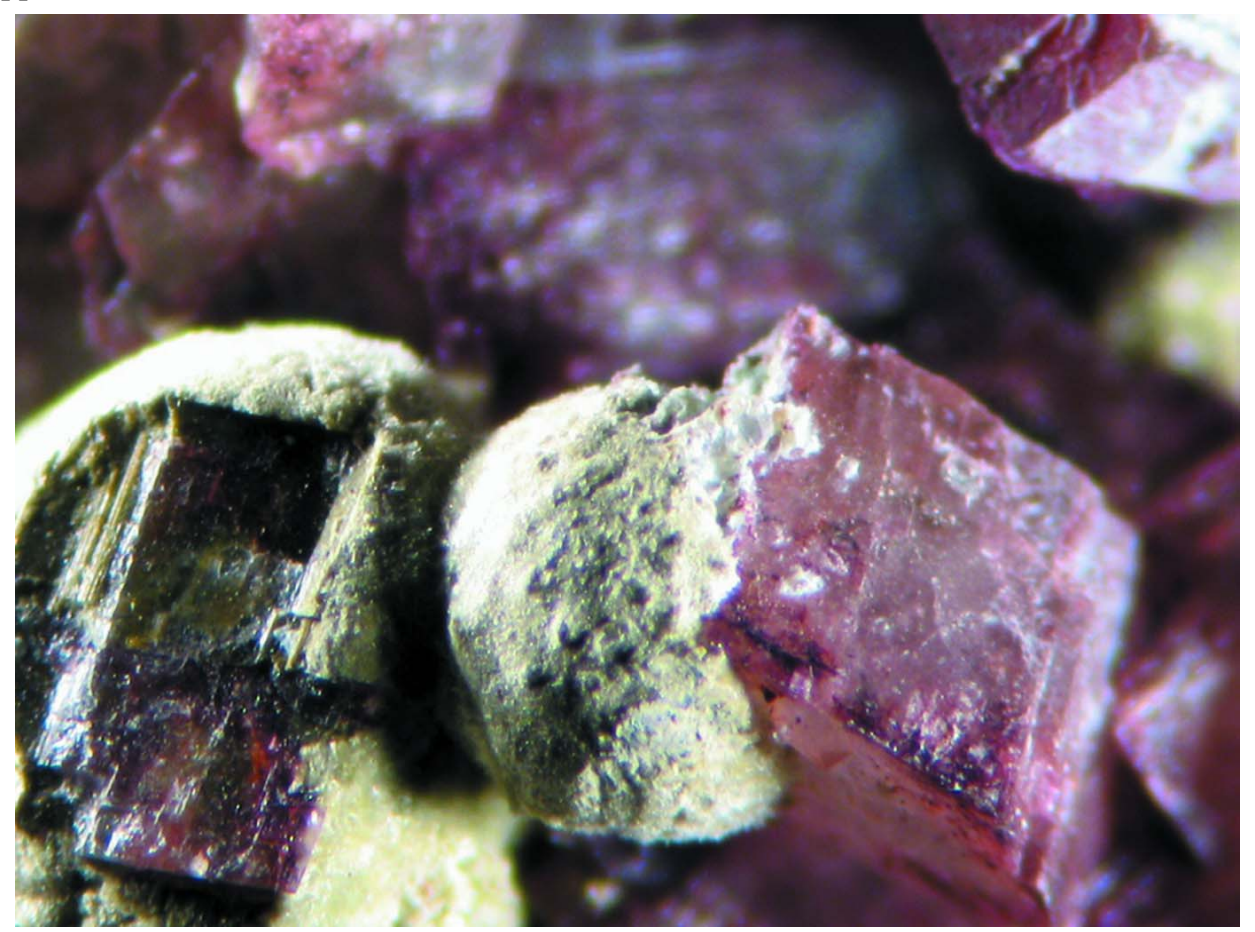

\section{Figure 1}

Micro photograph of the cobaltoan dolomite specimen, where pale pink cobaltoan dolomite is associated with pale green cobaltoan malachite. 


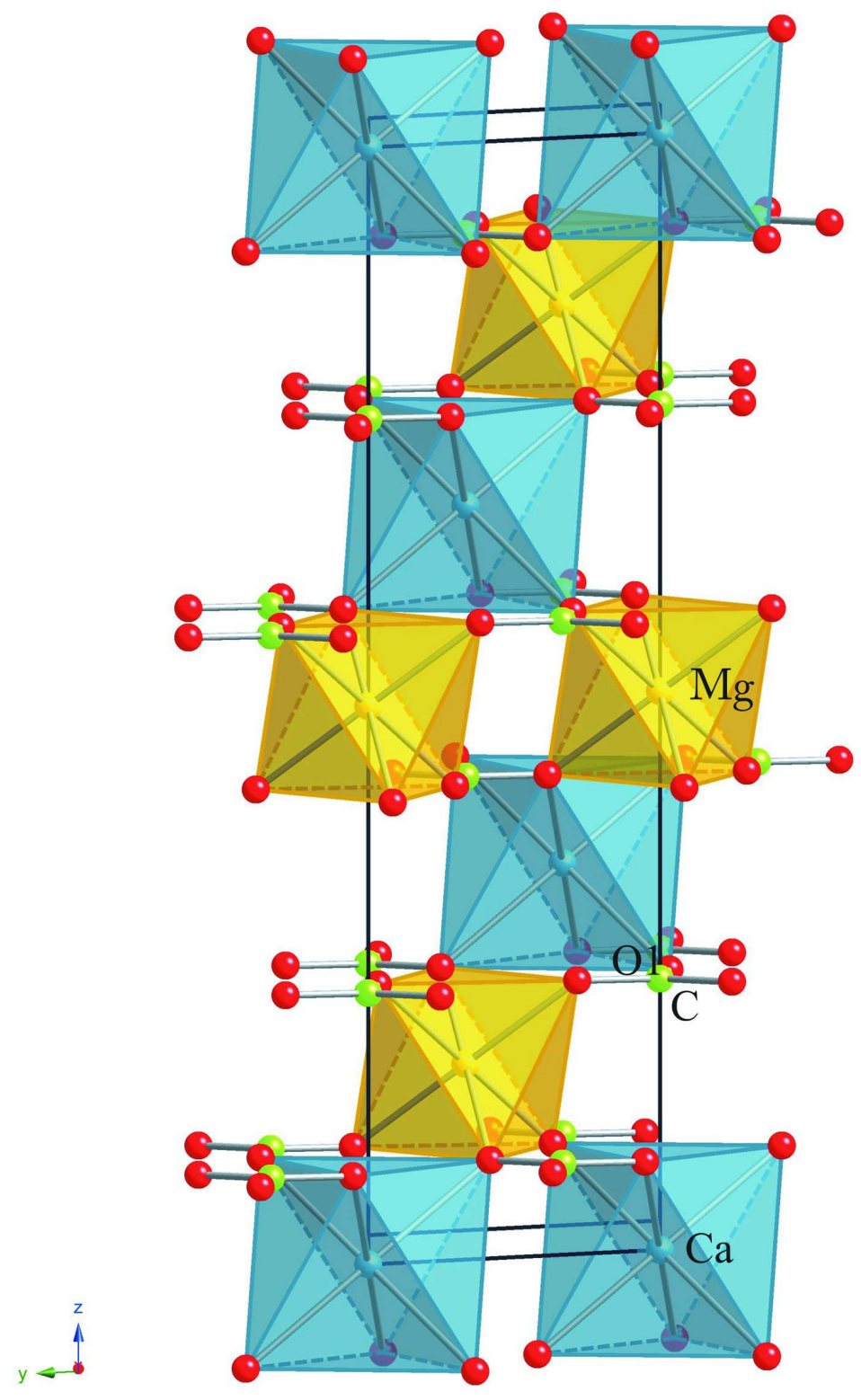

Figure 2

The crystal structure of cobaltoan dolomite, in a projection along [100], slightly tilted by $5^{\circ}$ about along the $x$ Cartesian rotation axis. Ca-centered octahedra are cyan, whereas $\mathrm{Mg}$-centered octahedra are yellow; carbon and oxygen atoms are represented as green and red spheres, respectively. 

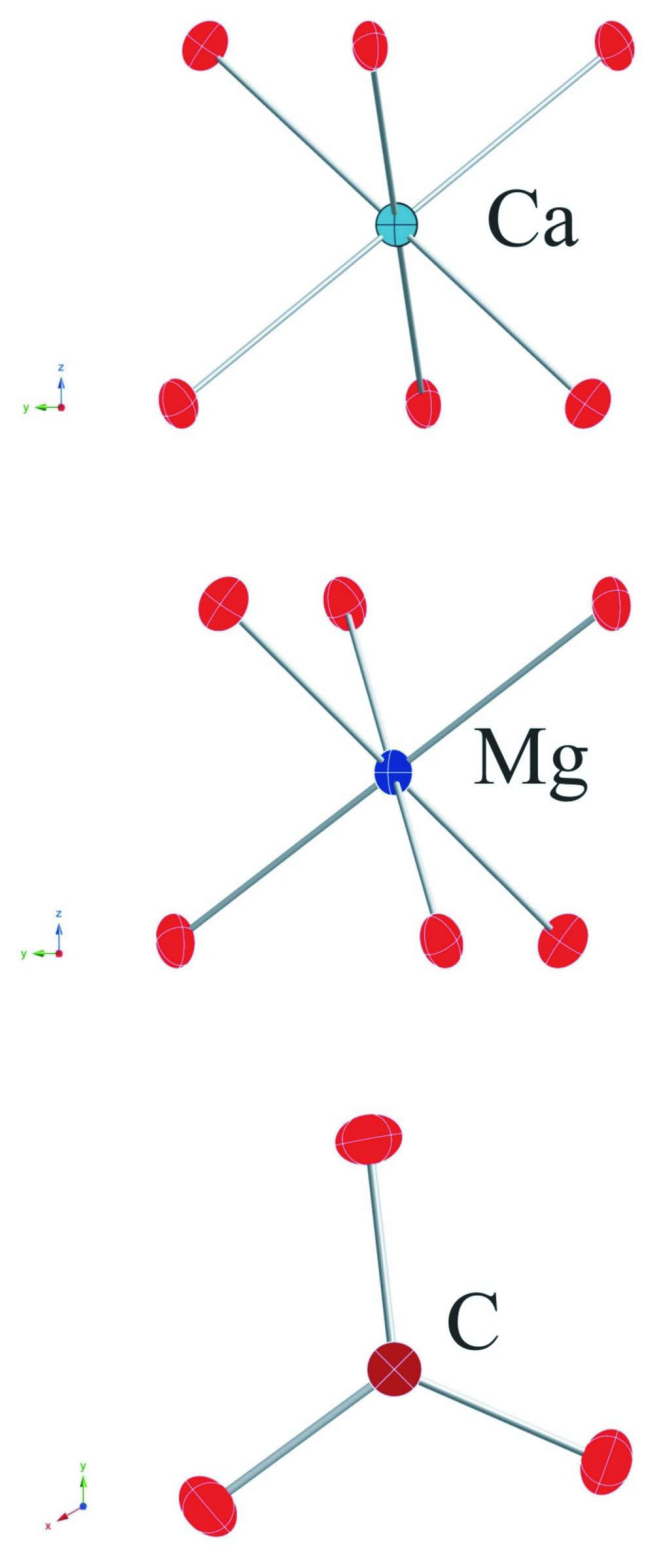

\section{Figure 3}

Coordination polyhedra in cobaltoan dolomite. Displacement ellipsoids are drawn at the 50\% probability.

\section{Calcium magnesium cobalt dicarbonate}

Crystal data

$\mathrm{CaMg}_{0.83} \mathrm{Co}_{0.17}\left(\mathrm{CO}_{3}\right)_{2}$

$M_{r}=190.38$

Trigonal, $R \overline{3}$

$a=4.8158(1) \AA$

$c=16.0488(6) \AA$

$V=322.34(2) \AA^{3}$

$Z=3$

$F(000)=284$

$D_{\mathrm{x}}=2.930 \mathrm{Mg} \mathrm{m}^{-3}$

Mo $K \alpha$ radiation, $\lambda=0.71073 \AA$

$\mu=2.17 \mathrm{~mm}^{-1}$

$T=295 \mathrm{~K}$

Cleavage rhombohedron, pale pink

$0.2 \times 0.15 \times 0.12 \mathrm{~mm}$ 


\section{Data collection}

Bruker SMART Breeze CCD

diffractometer

$\omega$ scans

Absorption correction: multi-scan

(SADABS; Bruker, 2008)

$T_{\min }=0.621, T_{\max }=0.746$

258 measured reflections

\section{Refinement}

Refinement on $F^{2}$

Least-squares matrix: full

$R\left[F^{2}>2 \sigma\left(F^{2}\right)\right]=0.019$

$w R\left(F^{2}\right)=0.059$

$S=0.96$

258 reflections

20 parameters
738 independent reflections

257 reflections with $I>2 \sigma(I)$

$R_{\text {int }}=0.010$

$\theta_{\text {max }}=32.4^{\circ}, \theta_{\text {min }}=3.8^{\circ}$

$h=-7 \rightarrow 3$

$k=0 \rightarrow 7$

$l=-23 \rightarrow 23$

1 restraint

$w=1 /\left[\sigma^{2}\left(F_{\mathrm{o}}{ }^{2}\right)+(0.0438 P)^{2}+0.562 P\right]$

where $P=\left(F_{\mathrm{o}}^{2}+2 F_{\mathrm{c}}^{2}\right) / 3$

$(\Delta / \sigma)_{\max }<0.001$

$\Delta \rho_{\max }=0.46 \mathrm{e} \AA^{-3}$

$\Delta \rho_{\min }=-0.32 \mathrm{e} \AA^{-3}$

\section{Special details}

Geometry. All e.s.d.'s (except the e.s.d. in the dihedral angle between two 1.s. planes) are estimated using the full covariance matrix. The cell e.s.d.'s are taken into account individually in the estimation of e.s.d.'s in distances, angles and torsion angles; correlations between e.s.d.'s in cell parameters are only used when they are defined by crystal symmetry. An approximate (isotropic) treatment of cell e.s.d.'s is used for estimating e.s.d.'s involving 1.s. planes.

Refinement. Refined as a 2-component twin.

Fractional atomic coordinates and isotropic or equivalent isotropic displacement parameters $\left(\hat{A}^{2}\right)$

\begin{tabular}{llllll}
\hline & $x$ & $y$ & $z$ & $U_{\text {iso }} * / U_{\text {eq }}$ & Occ. $(<1)$ \\
\hline Ca1 & 0.0000 & 0.0000 & 0.0000 & $0.01249(17)$ & \\
Mg1 & 0.0000 & 0.0000 & 0.5000 & $0.0104(3)$ & $0.828(4)$ \\
Co1 & 0.0000 & 0.0000 & 0.5000 & $0.0104(3)$ & $0.172(4)$ \\
C1 & 0.0000 & 0.0000 & $0.24297(12)$ & $0.0106(4)$ & \\
O1 & $0.2482(2)$ & $-0.0341(2)$ & $0.24403(6)$ & $0.0143(2)$ & \\
\hline
\end{tabular}

Atomic displacement parameters $\left(\AA^{2}\right)$

\begin{tabular}{lllllll}
\hline & $U^{11}$ & $U^{22}$ & $U^{33}$ & $U^{12}$ & $U^{13}$ & $U^{23}$ \\
\hline Ca1 & $0.0121(2)$ & $0.0121(2)$ & $0.0133(3)$ & $0.00605(10)$ & 0.000 & 0.000 \\
Mg1 & $0.0091(3)$ & $0.0091(3)$ & $0.0130(4)$ & $0.00456(15)$ & 0.000 & 0.000 \\
Co1 & $0.0091(3)$ & $0.0091(3)$ & $0.0130(4)$ & $0.00456(15)$ & 0.000 & 0.000 \\
C1 & $0.0103(5)$ & $0.0103(5)$ & $0.0112(8)$ & $0.0051(3)$ & 0.000 & 0.000 \\
O1 & $0.0117(4)$ & $0.0156(4)$ & $0.0183(4)$ & $0.0088(3)$ & $-0.0024(3)$ & $-0.0033(3)$ \\
\hline
\end{tabular}

Geometric parameters $\left(\AA,{ }^{\circ}\right)$

\begin{tabular}{llll}
\hline $\mathrm{Ca} 1-\mathrm{O} 1^{\mathrm{i}}$ & $2.3833(10)$ & $\mathrm{Mg} 1-\mathrm{O} 1^{\text {ix }}$ & $2.0923(9)$ \\
$\mathrm{Ca} 1-\mathrm{O} 1^{\text {ii }}$ & $2.3833(10)$ & $\mathrm{Mg} 1-\mathrm{O} 1^{\mathrm{x}}$ & $2.0923(9)$ \\
$\mathrm{Ca} 1-\mathrm{O} 1^{\mathrm{iii}}$ & $2.3833(10)$ & $\mathrm{Mg} 1-\mathrm{O} 1^{\mathrm{xi}}$ & $2.0923(9)$ \\
$\mathrm{Ca} 1-\mathrm{O} 1^{\text {iv }}$ & $2.3833(10)$ & $\mathrm{Mg} 1-\mathrm{O} 1^{\mathrm{xii}}$ & $2.0923(9)$ \\
$\mathrm{Ca} 1-\mathrm{O} 1^{\mathrm{v}}$ & $2.3833(10)$ & $\mathrm{C} 1-\mathrm{O} 1$ & $1.2853(9)$ \\
$\mathrm{Ca} 1-\mathrm{O} 1^{\mathrm{vi}}$ & $2.3833(10)$ & $\mathrm{C} 1-\mathrm{O} 1^{\mathrm{xiii}}$ & $1.2853(9)$
\end{tabular}




\begin{tabular}{|c|c|c|c|}
\hline $\mathrm{Mg} 1-\mathrm{O} 1^{\mathrm{vii}}$ & $2.0923(9)$ & $\mathrm{C} 1-\mathrm{O}^{\text {xiv }}$ & $1.2853(9)$ \\
\hline $\mathrm{Mg} 1-\mathrm{O} 1^{\text {viii }}$ & $2.0923(9)$ & $\mathrm{C} 1-\mathrm{Ca}^{\mathrm{xv}}$ & $3.1359(9)$ \\
\hline $\mathrm{O} 1^{\mathrm{i}}-\mathrm{Ca} 1-\mathrm{O} 1^{\mathrm{ii}}$ & $180.00(5)$ & $\mathrm{O} 1^{\mathrm{viii}}-\mathrm{Mg} 1-\mathrm{O} 1^{\mathrm{ix}}$ & $91.62(4)$ \\
\hline 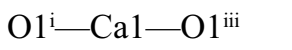 & $92.43(3)$ & $\mathrm{O} 1^{\mathrm{vii}}-\mathrm{Mg} 1-\mathrm{O} 1^{\mathrm{x}}$ & $91.62(4)$ \\
\hline 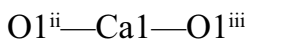 & $87.57(3)$ & $\mathrm{O} 1^{\mathrm{viii}}-\mathrm{Mg} 1-\mathrm{O}^{\mathrm{x}}$ & $88.38(4)$ \\
\hline $\mathrm{O} 1^{\mathrm{i}}-\mathrm{Ca} 1-\mathrm{O} 1^{\mathrm{iv}}$ & $87.57(3)$ & $\mathrm{O} 1^{\mathrm{ix}}-\mathrm{Mg} 1-\mathrm{O}^{\mathrm{x}}$ & 180.0 \\
\hline 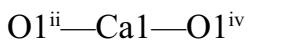 & $92.43(3)$ & 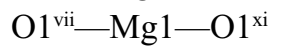 & $91.62(4)$ \\
\hline 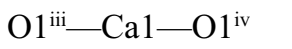 & $180.00(4)$ & $\mathrm{O} 1^{\mathrm{viii}}-\mathrm{Mg} 1-\mathrm{O} 1^{\mathrm{xi}}$ & $88.38(4)$ \\
\hline $\mathrm{O} 1^{\mathrm{i}}-\mathrm{Ca} 1-\mathrm{O}^{\mathrm{v}}$ & $87.57(3)$ & $\mathrm{O} 1^{\mathrm{ix}}-\mathrm{Mg} 1-\mathrm{O} 1^{\mathrm{xi}}$ & $91.62(4)$ \\
\hline $\mathrm{O} 1^{\mathrm{ii}-\mathrm{Ca} 1-\mathrm{O}^{\mathrm{v}}}$ & $92.43(3)$ & $\mathrm{O} 1^{\mathrm{x}}-\mathrm{Mg} 1-\mathrm{O} 1^{\mathrm{xi}}$ & $88.38(4)$ \\
\hline 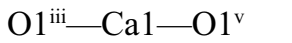 & $92.43(3)$ & $\mathrm{O} 1^{\mathrm{vii}}-\mathrm{Mg} 1-\mathrm{O} 1^{\mathrm{xii}}$ & $88.38(4)$ \\
\hline 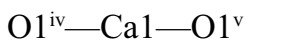 & $87.57(3)$ & 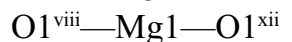 & $91.62(4)$ \\
\hline $\mathrm{O} 1^{\mathrm{i}}-\mathrm{Ca} 1-\mathrm{O} 1^{\mathrm{vi}}$ & $92.43(3)$ & $\mathrm{O} 1^{\mathrm{ix}}-\mathrm{Mg} 1-\mathrm{O} 1^{\mathrm{xii}}$ & $88.38(4)$ \\
\hline $\mathrm{O}^{\mathrm{ii}}-\mathrm{Ca} 1-\mathrm{O}^{\mathrm{vi}}$ & 87.57 (3) & $\mathrm{O} 1^{\mathrm{x}}-\mathrm{Mg} 1-\mathrm{O} 1^{\mathrm{xii}}$ & $91.62(4)$ \\
\hline $\mathrm{O} 1^{\mathrm{iii}}-\mathrm{Ca} 1-\mathrm{O} 1^{\mathrm{vi}}$ & $87.57(3)$ & $\mathrm{O} 1^{\mathrm{xi}}-\mathrm{Mg} 1-\mathrm{O} 1^{\mathrm{xii}}$ & $180.00(4)$ \\
\hline $\mathrm{O} 1^{\mathrm{iv}}-\mathrm{Ca} 1-\mathrm{O}^{\mathrm{vi}}$ & $92.43(3)$ & $\mathrm{O} 1-\mathrm{C} 1-\mathrm{O} 1^{\mathrm{xiii}}$ & $119.984(5)$ \\
\hline $\mathrm{O} 1^{\mathrm{v}}-\mathrm{Ca} 1-\mathrm{O} 1^{\mathrm{vi}}$ & $180.00(8)$ & $\mathrm{O} 1-\mathrm{C} 1-\mathrm{O} 1^{\mathrm{xiv}}$ & $119.983(5)$ \\
\hline 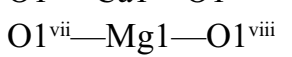 & 180.0 & $\mathrm{O} 1^{\mathrm{xiii}}-\mathrm{C} 1-\mathrm{O} 1^{\mathrm{xiv}}$ & $119.981(5)$ \\
\hline $\mathrm{O} 1^{\mathrm{vi}}-\mathrm{Mg} 1-\mathrm{O} 1^{\mathrm{ix}}$ & $88.38(4)$ & & \\
\hline
\end{tabular}

Symmetry codes: (i) $-x+y+1 / 3,-x+2 / 3, z-1 / 3$; (ii) $x-y-1 / 3, x-2 / 3,-z+1 / 3$; (iii) $-x+2 / 3,-y+1 / 3,-z+1 / 3$; (iv) $x-2 / 3, y-1 / 3, z-1 / 3$; (v) $-y+1 / 3, x-y-1 / 3$, $z-1 / 3$; (vi) $y-1 / 3,-x+y+1 / 3,-z+1 / 3$; (vii) $-x+y+2 / 3,-x+1 / 3, z+1 / 3$; (viii) $x-y-2 / 3, x-1 / 3,-z+2 / 3$; (ix) $-y-1 / 3, x-y-2 / 3, z+1 / 3$; (x) $y+1 / 3,-x+y+2 / 3$, $-z+2 / 3$; (xi) $-x+1 / 3,-y-1 / 3,-z+2 / 3$; (xii) $x-1 / 3, y+1 / 3, z+1 / 3$; (xiii) $-x+y,-x, z$; (xiv) $-y, x-y, z$; (xv) $x+2 / 3, y+1 / 3, z+1 / 3$. 